\title{
10-kV Electron-Diffractive Imaging of Multiwall Carbon Nanotube
}

\author{
O. Kamimura, ${ }^{* * *}$ T. Dobashi, ${ }^{*}$ K. Kawahara, ${ }^{* *}$ Y. Maehara, ${ }^{* *}$ and K. Gohara ${ }^{* *}$ \\ * Central Research Laboratory, Hitachi, Ltd., 1-280 Higashi-koigakubo Kokubunji-shi, Tokyo 185- \\ 8601, Japan \\ ** Division of Applied Physics, Graduate School of Engineering, Hokkaido University, Sapporo \\ 060-8628, Japan
}

Electron microscopes are often used to observe the atomic scale structure of materials. However, for the light element materials, e.g., carbon nanomaterials and organic semiconductors, specimen damage due to beam irradiation is a serious problem. Knock-on damage, which is significantly increased when a high-energy electron beam is used, is suppressed in the observation below the threshold energy. The threshold energy is specific to the specimen, depending on its component elements and the strength of their binding energy, e.g., about $27 \mathrm{keV}$ for carbon and $5 \mathrm{keV}$ for hydrogen $[1,2]$. Using a low-energy (low acceleration voltage) electron beam decreases the amount of knock-on damage. However, even at a low acceleration voltage, the ionization damage becomes significant. Moreover, low acceleration voltage causes various experimental difficulties, such as increasing of inelastic scattering and decreasing of the brightness of electron gun. Relating to these factors, we need to choice a suitable acceleration voltage for each specimen. Furthermore, lens aberrations mean that obtaining the atomic-resolution image at a low acceleration voltage is difficult. Meanwhile, diffractive imaging with iterative phase retrieval $[3,4]$ is one of the most promising techniques for high-resolution imaging. The object image is reconstructed from diffraction intensities by retrieving phases obtained from iteration procedures. This method is mainly used with X-rays [5-8] and published works on imaging done with the electron beam [9-13] have recently increased. To achieve high-resolution imaging without serious damage to the specimen, we verified the diffractive imaging with a relatively low-energy $(20 \mathrm{kV})$ electron beam [11], and developed an electron microscope that can be used for this method [14]. This microscope was based on the conventional scanning electron microscope, and a film loader system for the transmission electron microscope and a CCD camera system were installed to record the diffraction pattern without using a post-specimen lens (without projecting the back-focal plane of the objective lens). An additional function was the use of projection lenses to control the size of the diffraction pattern, i.e., camera length.

To enable this imaging to be used in a wider range of applications for radiation sensitive materials, we need to verify the diffractive imaging at a lower energy. We tried to record the diffraction pattern of a multiwall carbon nanotube (MWCNT) below an acceleration voltage of $20 \mathrm{kV}$. However, the sensitivity of the imaging plate (IP) of the electron microscope (Fujifilm FDL-UR-V) decreased rapidly below the $20 \mathrm{kV}$, and at $10 \mathrm{kV}$ it became almost unable to detect. We saw that the problem might be caused by the protective layer and selected the IP used for tritium detection (Fujifilm BAS-TR), which does not have such a layer, to record the lower voltage electron beam.

The diffraction pattern of the MWCNT at $10 \mathrm{kV}$ with $2048 \times 2048$ pixels is shown in Figure 1(a). The exposure time was $30 \mathrm{sec}$ and the geometrical convergence angle of illumination beam was estimated to be $0.15 \mathrm{mrad}$. The camera length was $453 \mathrm{~mm}$ by using a projection lens. The intensity distribution of the equatorial line (indicated by the arrows) in the diffraction pattern was clearly resolved. The beam size on the specimen was estimated to be about $100 \mathrm{~nm}$ mainly because of the diffraction aberration. The large illumination beam meant that some additional intensities from 
other nanotubes or graphite were also weakly recorded. The reconstructed pattern from the diffraction at $10 \mathrm{kV}$ is shown in Figure 1(b). In the reconstructed pattern, the characteristic features of a MWCNT with an inner and outer diameter of 2.5 and $4.5 \mathrm{~nm}$, respectively, as well as $0.34 \mathrm{~nm}$ carbon wall spacing can be observed, which is almost the same pattern as previously reported [11]. This finest feature reaches under 30 times of the wavelength $(30 \lambda)$, which is closely comparable to the values of the latest results of electron diffractive imaging [13] and results using a state-of-the-art aberration corrector. The newly developed electron microscope and phasing algorithm will also be presented.

\section{References}

[1] L. Reimer, Transmission Electron Microscopy, 3rd ed., Springer-Verlag, Berlin Heidelberg, 1993.

[2] R. Henderson, Q. Rev. Biophys. 28 (1995) 171

[3] R.W. Gerchberg and W. O. Saxton, Optik 35 (1972) 237.

[4] J. R. Fienup, Appl. Opt. 21 (1982) 2758.

[5] J. Miao et al., Nature 400 (1999) 342.

[6] D. Shapiro et al., Proc. Natl. Acad. Sci. USA 102 (2005) 15343.

[7] H. N. Chapman et al., Nat. Phys. 2 (2006) 839.

[8] Y. Nishino et al., Phys. Rev. Lett. 102 (2009) 018101.

[9] U. Weierstall et al., Ultramicroscopy 90 (2002) 171.

[10] J. M. Zuo et al., Science 300 (2003) 1419.

[11] O. Kamimura et al., Appl. Phys. Lett. 92 (2008) 024106.

[12] S. Morishita et al., Appl. Phys. Lett. 93 (2008) 183103.

[13] W. J. Huang et al, Nat. Phys. 5 (2009) 129.

[14] O. Kamimura et al., Microsco. Microanal. 14 (Suppl 2) (2008) 826.

[15] This research was partially supported by the Japan Science and Technology Agency (JST).
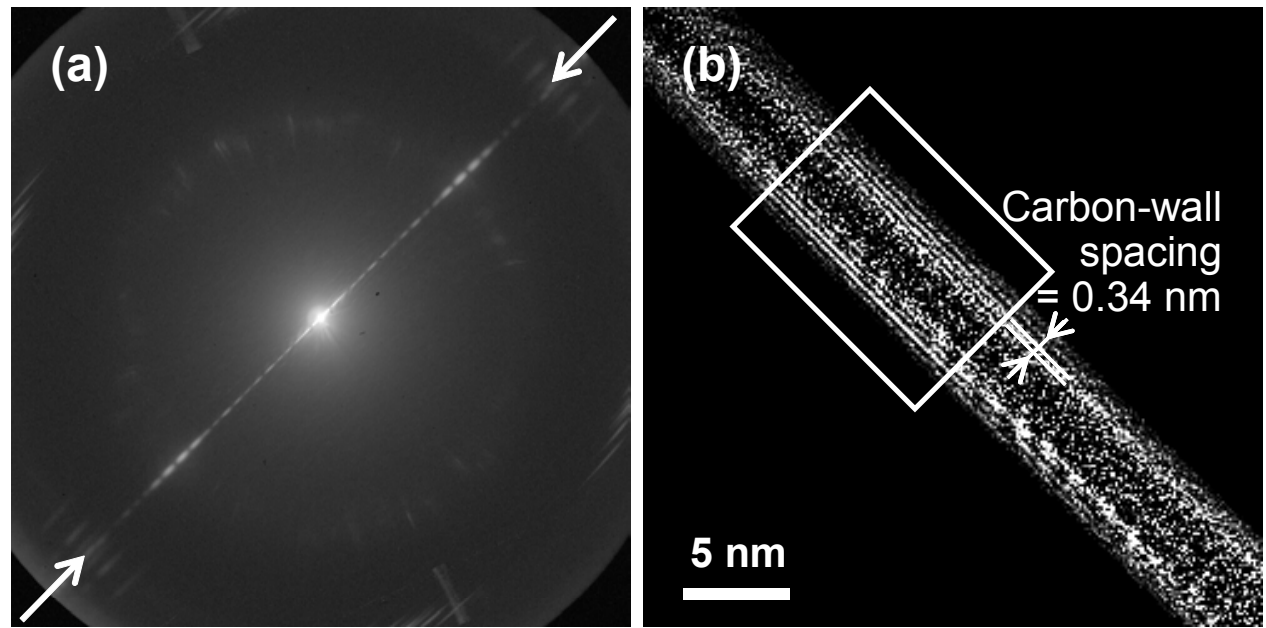

Fig.1. (a) Diffraction pattern of MWCNT at $10 \mathrm{kV}$ with 2048 × 2048 pixels. Exposure time was 30 sec. (b) Reconstructed pattern from diffraction pattern (a). Characteristic features of MWCNT as well as $0.34 \mathrm{~nm}$ carbon wall spacing were reconstructed especially in white box. 